\title{
Etude des chaleurs des vaches trypanotolérantes N'Dama et Baoulé en Côte d'Ivoire. II. Composante hormonale (LH)
}

\author{
C. Meyer ${ }^{1}$, P. Yesso ${ }^{2}$
}

MEYER (C.), YESSO (P.). Etude des chaleurs des vaches trypanotolérantes N'Dama et Baoulé en Côte d'Ivoire. II. Composante hormonale (LH). Revue Élev. Méd. vét. Pays trop., 1995, 48 (1) : 95-99

Les objectifs de cette étude sont, d'une part la détermination du délai de venue en astrus et la durée de celui-ci chez des femelles cycliques de deux races bovines (trypanotolérantes) N'Dama et Baoulé après administration de prostaglandine F2 $\alpha$ et, d'autre part, la mesure des caractéristiques périphériques concomitantes de l'hormone LH. L'étude a été conduite en saison sèche et chaude en zone tropicale humide (Bouaké, Côte d'Ivoire). Elle a porté sur 7 vaches N'dama et 8 Baoulé. Les vaches observées en chaleur l'ont été selon un délai moyen de 59,9 $\pm 9,2 \mathrm{~h}(7 \mathrm{~N}$ 'Dama) et $91,7 \pm 10,3 \mathrm{~h}$ (6 Baoulé) suivant l'injection de $15 \mathrm{mg}$ de luprostiol (analogue de la prostaglandine F2 $\alpha$ ). Le niveau de base moyen de l'hormone LH en dehors de la décharge préovulatoire était égal à $1,5 \pm 0,5 \mathrm{ng} / \mathrm{ml}$ (N'Dama) et 1,8 $\pm 0,7 \mathrm{ng} / \mathrm{ml}$ (Baoulé). Le pic préovulatoire de LH était typique sur 4 vaches N'Dama et 3 vaches Baoulé et présentait les caractéristiques suivantes : début 5,3 h après le début des chaleurs, durée 6-7 h, niveau maximal $60 \mathrm{ng} / \mathrm{ml}$. Ces observations suggèrent un délai de venue en chaleur significativement plus précoce de $30 \mathrm{~h}$ environ chez les N'Dama que chez les Baoulé après injection de prostaglandine F2 $\alpha$. Chez ces 2 races, pour les femelles qui ovulent, les concentrations circulantes de $\mathbf{L H}$ suivent des évolutions semblables à celles des bovins européens sous climat tempéré.

Mots clés : Vache - Bovin Baoulé - Bovin N'Dama - Taurin à courtes comes d'Afrique de l'Ouest - Reproduction - Cycle ostral - Prostaglandine - Hormone - Côte d'Ivoire.

\section{INTRODUCTION}

L'élevage des bovins de races trypanotolérantes présente un grand intérêt dans les pays où sévissent de façon endémique les trypanosomoses. Un des freins au développement de cet élevage est constitué par les faibles performances de reproduction de ces races en milieu villageois. La connaissance de la physiologie du cycle sexuel de leurs femelles est nécessaire pour raisonner les anomalies de la reproduction que l'on peut constater (20) et améliorer les performances de reproduction.

Les résultats de l'étude comportementale et anatomique au cours des chaleurs des vaches (trypanotolérantes) N'Dama et Baoulé ont été précédemment reportés (12). Le jour des chaleurs (déterminées avec l'aide de l'évolution de la progestérone plasmatique), 84,4 p. 100 des N'Dama et 89,3 p. 100 des Baoulé acceptent la monte.

1. CIRAD-EMVT, 10 rue Pierre Curie, 94704 Maisons-Alfort Cedex, France.

2. Institut des Savanes (IDESSA), Département Elevage, BP 1152, Bouaké, Côte-d'l'voire.

Reçu le 19.4.1994, accepté le 13.5.1995.
En effectuant deux observations de 30 min par jour, et en considérant comme critère des chaleurs au moins deux acceptations à la monte par jour, les deux tiers des chaleurs peuvent être ainsi détectés. Les caractéristiques des profils de $\mathrm{LH}$ chez les vaches des pays tempérés ont fait l'objet de nombreuses publications $(3,5,7,8,10,15$, $16,19,21,22,24,25,26)$. Ces études ont permis d'établir la chronologie des événements '(début et durée du pic de $\mathrm{LH}$ ) en fonction des chaleurs et de l'ovulation et d'expliquer pourquoi la période de fin des chaleurs est préférable pour inséminer.

Le but de la présente étude est d'évaluer le délai de venue en chaleur et la durée de l'œstrus après administration d'un agent lutéolytique (analogue de la prostaglandine $F 2 \alpha$ ) ainsi que d'établir l'évolution de la concentration de LH autour des chaleurs et ses relations chronologiques avec celles-ci chez les vaches de races locales ivoiriennes trypanotolérantes N'Dama et Baoulé.

\section{MATÉRIEL ET MÉTHODES}

\section{Matériel}

L'étude a eu lieu à la station centrale du Département des Ressources animales de l'IDESSA (Institut des Savanes) à Bouaké, dans le centre de la Côte d'Ivoire, au cours de la saison chaude et sèche (février). Elle a porté sur un lot de 7 vaches N'Dama et un autre de 8 vaches Baoulé adultes allaitantes pesant respectivement $268,9 \pm 31,2 \mathrm{~kg}$ (N'Dama) et 209,1 \pm 15,3 kg (Baoulé). Ces vaches ont toutes été reconnues cyclées auparavant par des observations journalières des chaleurs pendant plus de deux cycles œstraux. Elles étaient entretenues sur un pâturage de Panicum maximum variété $\mathrm{C} 1$. Un complément alimentaire composé de $4 \mathrm{~kg}$ de graines de coton et de $1 \mathrm{~kg}$ de mélasse par jour leur a été distribué tous les soirs, au parc de nuit où les vaches s'abreuvaient à volonté.

\section{Méthodes}

Les chaleurs de 15 vaches ont été synchronisées au moyen d'une injection intramusculaire de prostaglandine F2 $\alpha$ (15 mg de luprostiol $=$ Prosolvin $\left.{ }^{\circledR}\right)$ pratiquée le même jour à la même heure chez toutes les vaches qui se trouvaient alors entre le dixième et le quinzième jour de leur 
cycle œstral. La détection des chaleurs s'est faite à l'aide d'une vache androgénisée, présentée pendant une demiheure toutes les deux heures pendant 3 jours et 3 nuits en commençant 24 heures après l'injection. On a noté le nombre d'acceptations à la monte pour chaque demiheure d'observation. Ces données ont permis de déterminer le moment du début des chaleurs et la durée de cellesci pour chaque vache. Un examen gynécologique (vulve, utérus, ovaires) a été effectué avant et après l'étude.

Des prélèvements de sang ont été exécutés toutes les 2 heures durant la même période. Ils ont été effectués avec des tubes héparinés. Le sang a été centrifugé dans l'heure pour recueillir le plasma qui a été aussitôt congelé (9). La technique de dosage de l'hormone $\mathrm{LH}$ utilisée a été la méthode radioimmunologique décrite par Thibier (20). Le pic de LH a été défini comme l'ensemble des valeurs dépassant le niveau de base de LH augmenté de 3 fois son écart type (7).

\section{RÉSULTATS}

\section{Apparition et durée des chaleurs}

Deux vaches Baoulé n'ont pas accepté le chevauchement et n'ont donc pas été considérées pour le reste de l'étude. Chez les autres vaches, des acceptations de la monte ont été observées à partir de 59,9 $\pm 9,2 \mathrm{~h}$ (7 N'Dama) et $91,7 \pm 10,3 \mathrm{~h}$ ( 6 Baoulé) en moyenne après l'injection de prostaglandine $\mathrm{F} 2 \alpha$. La différence entre les délais moyens pour les deux races est hautement significative ( $p<0,001)$. L'apparition des chaleurs s'est échelonnée régulièrement, mais toutes les vaches N'Dama sont venues en chaleur avant les Baoulé (tabl. I). Certaines acceptations de monte ont été observées de façon très espacée avant ou après le pic des acceptations qui seul a été considéré pour le calcul de la durée des chaleurs. Celle-ci a été de 12,0 $\pm 5,4 \mathrm{~h}$ (N'Dama) et 10,3 \pm $6,5 \mathrm{~h}$ (Baoulé). Les variations sont grandes : on relève 1 à 9 séries d'acceptations séparées de 2 ou $4 \mathrm{~h}$ dans les pics d'acceptations. Une vache N'Dama et une Baoulé ont présenté des chaleurs durant moins de 2 heures.

\section{Profil de LH}

Le tableau II rapporte les caractéristiques observées pour ces vaches. Le pic de LH commençe tôt après le début des chaleurs $(2,5$ et $0,5 \mathrm{~h}$, respectivement chez les N'Dama et les Baoulé). II a une durée de 6 et 8,6 h respectivement chez les N'Dama et les Baoulé. II est typique et "pointu" sur 4 vaches N'Dama et 3 vaches Baoulé et commençe 4,75 $\pm 1,0$ h (N'Dama) ou 6,0 $\pm 6,0$ h (Baoulé) après le début des chaleurs. Son niveau maximal moyen est de $60 \pm 20,8 \mathrm{ng} / \mathrm{ml}$ (N'Dama) et de $57,7 \pm 5,8 \mathrm{ng} / \mathrm{ml}$ (Baoulé). En revanche, lorsqu'il est peu marqué ou tronqué (valeurs élevées avec un pic aplati), il commençe 0 (N'Dama) ou - 5 h (Baoulé) après le début des chaleurs, avec de plus grandes variations.
La figure 1 montre la courbe de LH type et le moment des manifestations des chaleurs d'une vache N'Dama qui présente des caractéristiques représentatives de ce groupe. Le début de l'augmentation de la décharge cyclique apparaît $67 \mathrm{~h}$ après l'injection de prostaglandine F2 $\alpha$. Le pic est atteint 3 à $4 \mathrm{~h}$ plus tard. La chute est forte et brutale. Le retour aux concentrations de base est observé $73 \mathrm{~h}$ après l'injection.

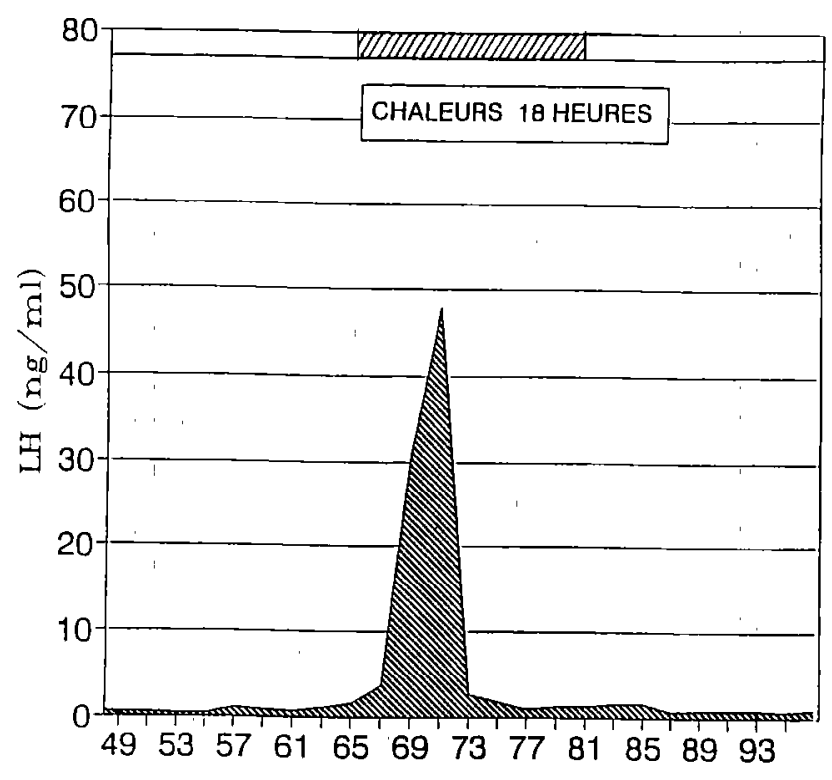

heures après injection de prostaglandine $F 2 \alpha$

Figure 1: LH autour des chaleurs chez une vache N'Dama.

\section{DISCUSSION}

L' œstrus des deux vaches Baoulé non vues en chaleur a pu être trop court, mais on aurait dû observer des valeurs élevées de LH. Or, le taux de LH était comparable au taux de base de la LH des autres vaches. Les chaleurs peuvent s'être manifestées au-delà de $119 \mathrm{~h}$ suivant l'injection, ce qui est rare. II est cependant vraisemblable qu'elles ont eu une décharge préovulatoire de $\mathrm{LH}$ et aient ainsi ovulé car un corps jaune a été observé 7 à 9 jours après les chaleurs des; autres vaches. Le poids de ces vaches était normal par rapport aux autres. Elles ne présentaient pas de misère physiologique ni de boiteries.

D'autres observations sur les mêmes races, concernant le délai d'apparition des chaleurs, ont donné des résultats semblables à ceux constatés dans cette étude pour les N'Dama mais distincts en race Baoulé (11). Les chaleurs étaient alors apparues 59,1 h (N'Dama) et 66,5 h (Baoulé) après l'injection de prostaglandine F2 $\alpha$. Toutefois, comme dans cet essai, en race Baoulé, Chicoteau (1) a observé la venue en chaleur $84,9 \pm 20,6 \mathrm{~h}$ après l'injection. 
Tableau I

Moment où les chaleurs ont été observées (jours et heures) après injection de prostaglandines

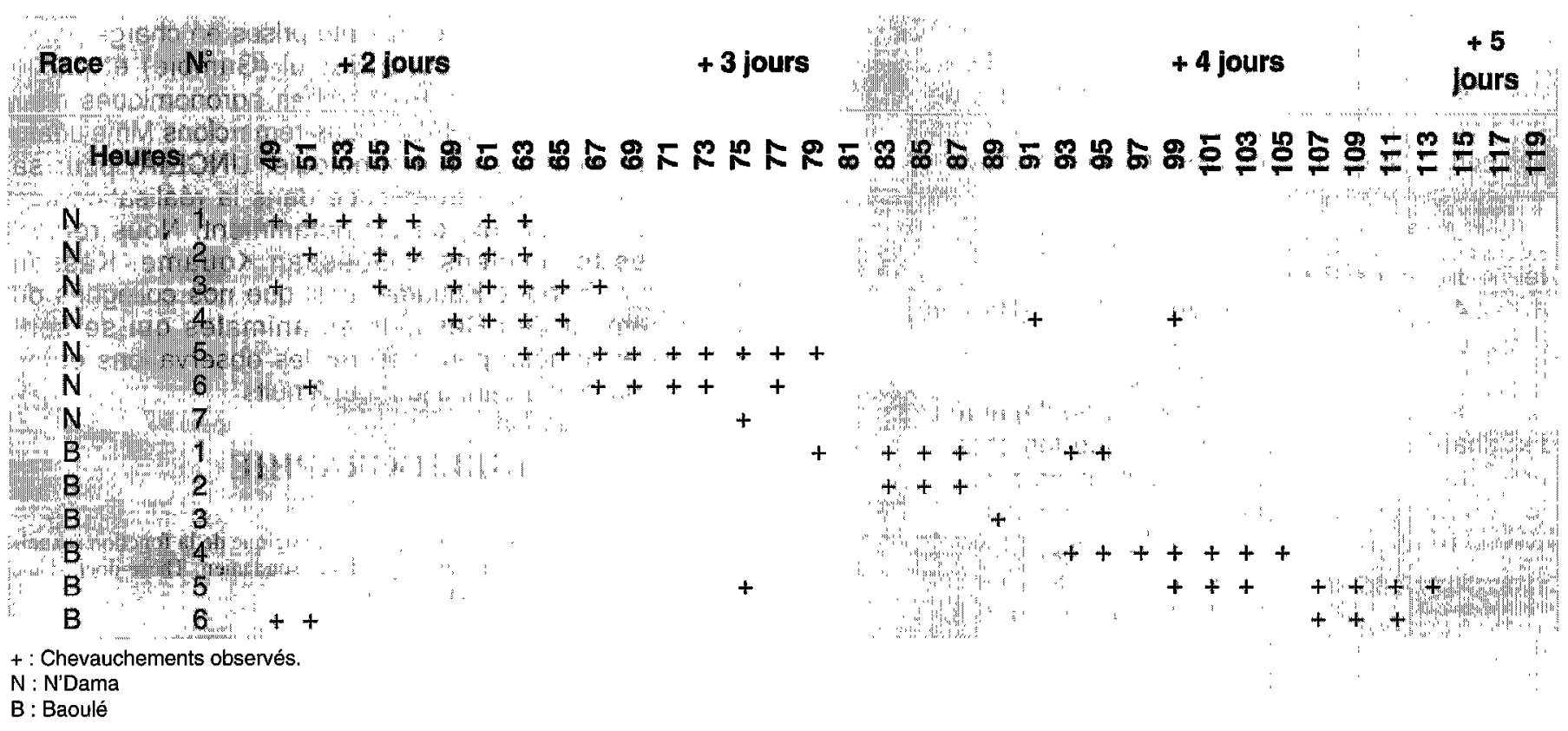

Tableau II

Caractéristiques moyennes du pic préovulatoire de la LH

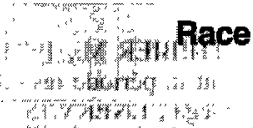

Durée des chaleurs $(h)$

Début des chaleurs - début

du pict L $(n)$,

Duree dupial $\mathrm{H}(\mathrm{h})$

Niveat maxtinal moyen du

pic LH (ng/N)

Niveau de base moyen de LH (ng $/ \mathrm{ml})$

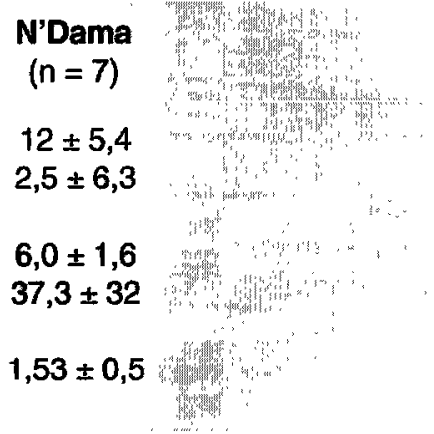

(*) 2 vaches Baoulé sur les 8 initiales n'ont pas eu de pic de LH pendant la période de prélèvements.

La durée des chaleurs observée ici après synchronisation au moyen de prostaglandine F2 $\alpha$ est semblable à celle des chaleurs naturelles dans les deux races : 8 à $9 \mathrm{~h}$ (15) ou $9,38 \pm 1,51 \mathrm{~h}(22)$ en race N'Dama et $10,7 \pm 5,1 \mathrm{~h}(1)$ en race Baoulé. Chez les vaches de races européennes (non trypanotolérantes), la durée des chaleurs est généralement plus longue que celle des races locales : 14-16h (6). Cette différence est liée à d'autres facteurs que la trypanotolérance puisqu'elle s'observe aussi chez les zébus (non trypanotolérants) vivant dans le même milieu que les taurins N'Dama et Baoulé (14).

Le pic de LH commence environ 6 heures (3-7 h) après le début des chaleurs chez les vaches européennes (3, $5,7,8,10,15,16,19,21,22,24,25,26)$. La présente observation révèle des valeurs semblables lorsque le pic de LH est typique. Chez la vache européenne (mêmes références), il dure 6 à $12 \mathrm{~h}$. Les présents résultats sont aussi situés dans ces limites. Le niveau maximal rapporté du pic de LH est de 5 à $100 \mathrm{ng} / \mathrm{ml}$ chez les vaches européennes et selon les systèmes de dosage ou les standards utilisés. Lorsque le pic est bien marqué, et dans le système utilisé ici, il est de $60 \mathrm{ng} / \mathrm{ml}$ environ en races N'Dama et Baoulé. Chicoteau et al. (2) ont relevé un niveau de $100 \mathrm{ng} / \mathrm{ml}$ en race Baoulé en effectuant des prises de sang toutes les heures et en ayant recours au même système de dosage, au même laboratoire. Cómme elles ont été effectuées toutes les deux heures dans le cas présent, le pic réel a pu se trouver entre deux prélèvements. Le niveau de base de l'hormone LH est de l'ordre de 1 à $2 \mathrm{ng} / \mathrm{ml}$ chez les bovins européens. Les résultats de cette étude sont de même grandeur. Chicoteau et al. (2) ont observé, en race Baoulé, que le pic de LH présente des caractéristiques proches de celui des races européennes. Cette étude, conduite dans une région différente, confirme cette observation et montre qu'il en est de même pour la race N'Dama. 


\section{Meyer P. Yesso}

On peut se demander si les chaleurs étudiées ici étaient normales chez toutes les vaches avec pic de $\mathrm{LH}$ typique ou non. A la suite de l'étude, 7 à 9 jours après les chaleurs, un ou deux corps jaunes palpables ont été décelés, aussi bien parmi les vaches présentant un pic de LH normal que parmi celles présentant un pic tronqué comme d'ailleurs celles qui n'ont pas accepté le chevauchement (cf. supra.).

Si l'on admet que l'ovulation survient 20 à $24 \mathrm{~h}$ après le début du pic de $\operatorname{LH}(8,10,13,18)$, celui-ci commençant vers $64,7 \mathrm{~h}$ (N'Dama) et vers $97,7 \mathrm{~h}$ (Baoulé) après l'injection, l'ovulation surviendrait vers $86,7 \mathrm{~h}$ (N'Dama) et vers $119,7 \mathrm{~h}$ (Baoulé) après l'injection de prostaglandine F2 $\alpha$, soit $26,8 \mathrm{~h}$ (N'Dama) et $28 \mathrm{~h}$ (Baoulé) après le début des chaleurs. On peut admettre que les chaleurs commencent vers $90 \mathrm{~h}$ après l'injection de prostaglandine $\mathrm{F} 2 \alpha$ en race Baoulé (Chicoteau cite $88 \mathrm{~h}$ et il a été trouvé ici $91,7 \mathrm{~h}$ ) et vers $60 \mathrm{~h}$ chez les N'Dama. Elles s'observeraient ainsi de 60 à $72 \mathrm{~h}$ (N'Dama) et de 90 à $100 \mathrm{~h}$ environ (Baoulé) après l'injection. Sachant que le meilleur moment pour inséminer correspond à la deuxième moitié des chaleurs chez les bovins européens, et si cela est vrai aussi dans ces races, ce moment serait entre 66 et $72 \mathrm{~h}$ (N'Dama) et entre 95 et $100 \mathrm{~h}$ (Baoulé) après l'injection de prostaglandine $\mathrm{F} 2 \alpha$. Ce moment mérite d'être précisé sur un effectif plus grand. Si ces observations originales se trouvaient pleinement confirmées, ceci constituerait un modèle physiologique original pour élucider le mécanisme de déclenchement de l'acceptation du chevauchement en raison de différences génétiques de cette ampleur, en milieu identique. Toutefois, cette constatation ne semble pas due à la faiblesse de l'effectif des vaches de cet essai car le centre d'insémination artificielle de Bingerville (Côte d'Ivoire) semble déjà tirer avantage d'inséminations plus précoces de $24 \mathrm{~h}$ après synchronisation des chaleurs avec des analogues de prostaglandine $\mathrm{F} 2 \alpha$ chez des femelles de race N'Dama qu'en race Baoulé (Galet, communication personnelle). Les résultats de l'essai viennent conforter cette pratique.

\section{CONCLUSION}

Les pics de LH pendant les chaleurs chez les vaches (trypanotolérantes) N'Dama et Baoulé présentent généralement des caractéristiques voisines de celles des races européennes. Les vaches N'Dama viennent plus précocement en chaleur que les Baoulé après synchronisation des chaleurs avec la prostaglandine F2 $\alpha$. En admettant qu'il est indiqué d'inséminer ces vaches dans la deuxième moitié des chaleurs naturelles, et que le moment d'apparition des chaleurs après injection de prostaglandine F2 $\alpha$ est proche de $60 \mathrm{~h}$ en race N'Dama et de $90 \mathrm{~h}$ en race Baoulé, le meilleur moment pour inséminer se situerait entre 66 et $72 \mathrm{~h}$ (N'Dama) et entre 95 et $100 \mathrm{~h}$ (Baoulé) après cette injection, soit en moyenne une différence de $28,5 \mathrm{~h}$ entre les deux races.

\section{REMERCIEMENTS}

Les analyses d'hormones ont été prises en charge par le projet FAO/RAF/88/100, à Banjul (Gambie) et par le Centre international de Recherches agronomiques pour le Développement (CIRAD). Nous remercions Mme Jeanguyot (Laboratoire d'hormonologie, UNCEIA) pour sa contribution aimable et efficace dans la réalisation des dosages hormonaux, de LH notamment. Nous remercions les techniciens N'Guessan Kouame, Kassou Kouassi et Yoboue Kouame ainsi que nos collègues du Département des Ressources animales qui se sont relayés avec nous pour assurer les observations et les prélèvements pendant 3 jours et 3 nuits.

\section{BIBLIOGRAPHIE}

1. CHICOTEAU P.,1989. Adaptation physiologique de la fonction sexuelle des bovins Baoulé au milieu tropical sud-soudanien. Thèse doct., Université Paris XII, Créteil, France, 174 p.

2. CHICOTEAU P., HUMBLOT P., CLOE C., BASSINGA A., THIBIER M., 1989. Physiological and pathological plasma profiles of progesterone and LH in indigenous West African Shorthorn cows (Bos taurus taurus). Zuchthyg., 24: 193-200.

3. CHRISTENSEN D.S., HOPWOOD M.L., WILTBANK J.N., 1974. Level of hormones in the serum of cycling beef cows. J. Anim. Sci., 38 (3): $577-583$.

4. DESSOUTER C., DENIS J.P., PAREZ M., THIBIER M., 1983. Profil des hormones gonadotropes (FSH et LH) pendant la période cestrale chez une femelle zébu pakistanaise. Dakar-Hann, Sénćgal, LNERV/ISRA, septembre 1983, 6 p. (n ${ }^{\circ}$ 64/Zoot)

5. DIELEMAN S.J., BEVERS M.M., VAN TOL H.T.M., WILLEMSE A.H., 1986. Peripheral plasma concentrations of oestradiol, progesterone, cortisol, $\mathrm{LH}$ and prolactin during the oestrous cycle in the cow, with emphasis on the peri oestrous period. Anim. Prod. Sci., 10: 275-292.

6. DOBSON H., KAMONTAPANA M., 1966. A review of female cattle reproduction with special reference to a comparison between buffaloes, cows and zebu. J. Reprod. Fert., 77: 1-36.

7. GAUTHIER D., 1986. The influence of season and shade on oestrus behaviour, timing of preovulatory $\mathrm{LH}$ surge and the pattern of progesterone secretion in 'FFPN and creole heifers in tropical climate. Reprod. 'Nutr. Dev., 26 (3): 767-775.

8. HENRICKS D.M., DICKEY F.J., NISWENDER G.D., 1970. Serum LH and plasma progesterone levels during the estrous cycle and early pregnancy in cows. Biol. Reprod., 2: 346-351.

9. HUMBLOT P., THIBIER M., 1984. Evaluation comparée des méthodes de diagnostic de gestation chez les bovins. Elevage Insém., $200: 3-18$.

10. KARG H., Recent results concerning cyclic effects of pituitary gonadotropins. In: VIIle congrès international de Reproduction animale et d'insémination artificielle, Munich, Allemagne, 6-9 juin 1972, Vol. I. p. 46-52.

11. MEYER C., YESSO P., 1990. Moment de l'apparition des chaleurs après injection de prostaglandines en races bovines (trypanotolérantes) N'Dama et Baoulé. Bouaké, Côte-d'Ivoire, IDESSA/DE - ZOOT, octobre 1990,13 p. (Note technique)

12. MEYER C., YESSO P., 1991. Les chaleurs des vaches trypanotolérantes Ndama et Baoulé en Côte-d'Ivoirc. I. Particularitćs des composantes comportementale et organique. Revue Élev. Méd vét. Pays trop., 44 (2) : 199-206. 
13. MIKESKA J.C., WILLIAMS G.L., 1988. Timing of preovulatory endocrine events. Estrus and ovulation in Brahman $x$ Hereford females synchronized with Norgestomet and estradiol valerate. J. Anim. Sci., 66: 939-946.

14. MUKASA-MUGERWA E., 1989. A review of reproductive performance of female Bos indicus (zebu) cattle. Addis-Abeba, Ethiopic, ILCA, 134 p. (Monograph no. 6)

15. PETERS A.R., 1985. Studies of hormone patterns during the oestrous cycle of beef cows. Reprod. Nutr. Dev., 25 (5) : 919-927.

16. RAHE C.H., OWENS R.E., FLEEGER J.L., NEWTON H.J., HARMS P.G., 1980. Pattern of plasma LH in the cyclic cow: dependance upon the period of the cycle. Endocrinology, 107 (2): 498-503.

17. RALAMBOFIRINGA A., 1978. Notes sur les manifestations du cycle cestral et sur la reproduction des femelles N'Dama. Revue Elev. Méd. vét. Pays trop., 31 (1) : 91-94.

18. RANDEL D.R., 1984. Reproductive endocrinology of Brahman cattle. In: Chemineau P., Gauthier D., Thimonier J. éds, Reproduction des ruminants en zone tropicale. Actes de la réunion internationale, Pointe-à-Pitre, Guadeloupe, France, 8-10 juin 1983. Paris, France, INRA, p. 159-188. (Coll. Les colloques de l'INRA $n^{\circ} 20$ )

19. SCHAMS D., SCHALLENBERGER E., HOFFMANN B., KARG H., 1987. The nestrous cycle of the cow : hnrmonal parameters and time relationships concerning oestrus, ovulation, and electrical resistance of the vaginal mucus. Acta Endocr., 86: 180-192.

MEYER (C.), YESSO (P.). Study of oestrus of N'Dama and Baoulé trypanotolerant cows in Côte d'Ivoire. II. Hormonal component (LH). Revue Élev. Méd. vét. Pays trop., 1995, 48 (1): $95-99$

The aims of this study were to evaluate the time elapsed between the injection of prostaglandin $F 2 \alpha$ and the onset of oestrus (and the duration of oestrus) of cows from two Bos taurus (trypanotolerant) breeds: N'Dama and Baoulé (West African shorthorn) and to measure the plasma peripheral concentration of LH around uestrus. The study was performed in a humid tropical zone (Bouaké, Côte d'Ivoire) during the dry hot season from 7 N'Dama and 8 Baoulé cycling cows. The onset of oestrus of the cows was observed $59.9 \pm 9.2$ hours (7 N'Dama) and $91.7 \pm 10.3 \mathrm{~h}$ (6 Baoulé) after injection with $15 \mathrm{mg}$ of luprostiol (prostaglandin F2 $\alpha$ analog). The base level of LH was 1.5 $\pm 0.5 \mathrm{ng} / \mathrm{ml}$ (N'Dama) and $1.8 \pm 0.7 \mathrm{ng} / \mathrm{ml}$ (Baoulé). The $\mathrm{LH}$ peak was typical in 4 N'Dama and 3 Baoulé cows and had the following parameters : beginning of the surge $5.3 \mathrm{~h}$ after onset of oestrus, duration 6-7 h, maximum level $60 \mathrm{ng} / \mathrm{ml}$. The two main features of this study were therefore: the onset of oestrus began significantly earlier ( $30 \mathrm{~h}$ approximately) in N'Dama than in Baoulé cattle after injection of prostaglandin F2 $\alpha$; the peripheral LH concentrations from these two breeds appeared to have a similar evolution to that of European cattle in temperate climates.

Key words : Cow - Baoulé cattle - N'Dama cattle - West African shorthorn cattle - Reproduction - Oestrous cycle - Prostaglandin - Hormone Côte d'Ivoire.
20. THIBIER M., 1975. Variations circadiennes de la LH plasmatique chez le jeune taurillon. Annls Endocr., 36 : 205-210.

21. TIIIBIER M., 1976. Les prostaglandines. Le cycle sexuel des mammifères domestiques. I. Description du cycle sexuel de la vache. Écon. Méd. anim., 17 (3) : 116-134

22. THIBIER M., HUMBLOT P., 1981. L'utérus et le cycle sexuel (chap. II). In: Constantin A., Meissonnier E., éds, L'utérus de la vache. MaisonsAlfort, France, Soc. fr. Buiatrie, ENVA, p. 53-78.

23. TRAORE A., BAKO G., 1984. Etude du cycle sexuel chez les vaches et génisses N'Dama élevées au Centre de Recherches Zootechniques de Sotuba (Mali). I. Incidence de l'utilisation d'un taureau boute-en-train sur le taux de détection des chaleurs. Revue Élev. Méd. vét. Pays trop., 34 (4): $482-484$.

24. VAISSAIRE J.P., 1977. Sexualité et reproduction des mammifères domestiques et de laboratoire. Paris, France, Maloine éd., 457 p.

25. WALTERS D.L., SCHALLENBERGER E., 1984. Pulsatile secretion of gonadotrophins, ovarian steroids and ovarian ocytocin during the periovulatory phase of the oestrous cycle in the cow. J. Reprod. Fert., 71: 503-512.

26. WALTON J.S., VEENHUIZEN L.P., KING G.J., 1987. Relationships between time of day, estrous behaviour, and the preovulatory LH surge in Holstein after treatment with cloprostenol. J. Dairy Sci., 70: 1652-1663.

27. WETTEMANN R.P., HAFS H.D., EDGERTON L.A., SWANSON L.V., 1972. Estradiol and progesterone in blood serum during the bovine estrous cycle. J. Anim. Sci., 34 (6): 1020-1024.

MEYER (C.), YESSO (P.). Estudio de los celos en las vacas tripanotolerantes N'Dama y Baulé en Côte d'Ivoire. II. Componente hormonal (LH). Revue Élev. Méd. vét. Pays trop., 1995, 48 (1) : 95-99

Los objetivos del presente estudio son, por un lado, la determinación del intervalo de retorno a estro y de la duración de éste en hembras adultas de dos razas bovinas (tripano-tolerantes) N'Dama y Baulé, post administración de prostaglandinas $F 2-\alpha$ y por otro lado, la medida de las características periféricas concomitantes de la hormona LH. El estudio se llevó a cabo durante la estación seca y caliente en una zona tropical húmeda (Bouaké, Côte d'Ivoire), en 7 vacas N'Dama y 8 Baulé. La duración de los celos obscrvados en las hembras fue de $59,9 \pm 9,2$ horas ( 7 N'Dama) y de $91,7 \pm 10,3$ h (6 Baulé), post inyección de $15 \mathrm{mg}$ de luprostiol (análogo de prostaglandina F2$\alpha$ ). El nivel básico medio de la hormona LH, fuera de la descarga pre ovulatoria, fue igual a $1,5 \pm 0,5 \mathrm{ng} / \mathrm{ml}$ (N'Dama) y a $1,8 \pm 0,7$ $\mathrm{ng} / \mathrm{ml}$ (Baulé). El pico pre ovulatoria de LH fue típico en 4 vacas N'Dama y 3 vacas Baulé, presentando las siguientes características: inicio 5,3 horas post comienzo del celo, duración de 6-7 h, nivel máximo de $60 \mathrm{ng} / \mathrm{ml}$. Estas observaciones sugieren un intervalo de retorno a celo significativamente precoz en las vacas N'Dama, de 30 horas post unyección de prostanglandinas $F 2-\alpha$. En estos dos animales, al menos en las hembras que ovulan, las concentraciones de LH circulantes siguieron una evolución similar a la de los bovinos europeos bajo clima templado.

Palabras clave : Vaca - Bovino Baulé - Bovina N'Dama - Bovino de cuemos cortos de Africa occidental - Reproducción - Ciclo estral - Prostaglandina - Hormona - Côte d'Ivoire. 